\title{
Study on the Distributed Parameter Model of the Billet Induction Heating Process Based on the Finite Difference Method
}

\author{
$\mathrm{Xu}$ Zhe \\ Information and control institute \\ Hangzhou Dianzi University \\ Hangzhou,China \\ xuzhe@hdu.edu.cn \\ Fang Dong \\ Information and control institute \\ Hangzhou Dianzi University \\ Hangzhou,China \\ endness@126.com
}

\author{
Che Xulong \\ Information and control institute \\ Hangzhou Dianzi University \\ Hangzhou,China \\ chexvlong1988@126.com \\ Kong Yaguang \\ Information and control institute \\ Hangzhou Dianzi University \\ Hangzhou,China \\ ygkong@hdu.edu.cn
}

\begin{abstract}
In this paper a distributed parameter model of the continuous casting billet induction heating process is established. It uses the finite difference method to solve the two-dimensional temperature field of the billet cross-section, predicts the variation of the billet temperature distribution during heating, and verifies the results with ANSYS finite element simulation data. The results show that this method can achieve high prediction accuracy, and is helpful to achieve precise control of the billet temperature in induction heating process.
\end{abstract}

Keywords-Finite difference; Distributed parameter model; Induction heating

\section{INTRODUCTION}

Induction heating has many advantages, such as high speed of heating, not easy oxidation and decarbonization, green energy, easy to control, etc, so the technique have been widely used in steel metallurgy and other industrial production. However, since the surface of the billet is rapidly heated by the skin effect, many physical effects including heating, radiating and heat conduction happen simultaneously during the billet moving, and the total temperature field of the billet changes not only with time but space, the billet induction heating process belongs to the typical distributed parameter system.

Due to the limitation of the current temperature measurement technology, it is unable to measure the internal temperature of the billet during the induction heating process. In order to obtain the temperature distribution inside the billet, a mathematic model of the billet should be established to achieve a soft measurement.

In the study of the mathematic model of the billet induction heating, B.S. Jovanovic realized the calculation of the temperature field during the heat conduction through the finite difference method [1]. Xue Qiong used the finite volume method to analyze and calculate the onedimensional and two-dimensional heat conduction problems [2]. Liu Hao solved the problem by introduction of the complex magnetic potential vector and complex electric potential vector to calculate the Joule heat generated in the billet induction heating process [3].

The paper establishes a distributed parameter model of the billet induction heating process. It solves the twodimensional temperature field of the billet cross-section through the finite difference method, then verifies the results of the billet temperature distribution with the finite element analysis software ANSYS simulation data, and obtains the variation law of the temperature field of the billet induction heating process.

\section{DISTRIBUTED PARAMETER MATHEMATICAL MODEL ANALYSIS}

The model takes one billet internal micro unit as a research object, analyzes the variation law of its temperature through heat transfer mechanism, and then deduces the multi-dimensional temperature field of partial differential equation of the billet heating process [4].

In the billet induction heating process, according to Fourier Theorem and the principle of conservation of energy on the surface, heat conduction equation is obtained as follows:

$$
\rho c \frac{\partial T}{\partial t}=\lambda\left(\frac{\partial^{2} T}{\partial x^{2}}+\frac{\partial^{2} T}{\partial y^{2}}+\frac{\partial^{2} T}{\partial z^{2}}\right)+q_{v}
$$

Where $\rho$ is density, $c$ is specific heat, $\lambda$ is thermal conductivity, $q_{v}$ is the rate of heat generation density of the heat source.

Initial conditions: the initial temperature of the billet is the billet temperature first into furnace.

Boundary conditions: heat transfer on the surface of the billet and the surroundings is mainly in manner of convection and radiation. Since convection is weak with respect to radiation when the temperature is very high, this paper ignores the influence of convection. The density of radiation heat transfer is

$$
q_{r}=\varepsilon \sigma\left(T_{w}^{4}-T_{s u r}^{4}\right)
$$


Where $T_{w}$ is the surface temperature of the billet, $T_{\text {sur }}$ is the ambient temperature, $\varepsilon$ is emissivity, $\sigma$ is a blackbody radiation constant.

\section{FINITE ELEMENT CALCULATION OF THE DISTRIBUTED PARAMETER MODEL}

A three-dimensional temperature control equation (1) of the billet cross-section in induction heating process is obtained above. Some necessary assumptions should be made to ensure the rationality of the control equation in solving practical problem. The paper makes the following assumptions according to the situation of the induction heating process.

- The heat during induction heating is primarily generated by eddy current, so heat conduction of the billet in the direction of the furnace length is ignored;

- The billet exchanges heat with the environment by thermal radiation from the upper and lower, left and right surfaces in the moving process. Each side has the same heat conduction, so the study object is simplified to $1 / 8$ of the billet cross-section. A total of 15 nodes are shown in boxed section of Fig. 1;

- According to the skin effect, eddy is mainly produced on the surface of the billet, so the eddy current power decreases from the surface to the inside.

- The billet surface oxidation on the temperature distribution is ignored;

- End effect of heat conduction is ignored;

- The billet is uniformly heated.

$$
\rho c \frac{\partial T}{\partial t}=\lambda\left(\frac{\partial^{2} T}{\partial x^{2}}+\frac{\partial^{2} T}{\partial y^{2}}\right)+q_{v}
$$

To establish a distributed parameter model:

\section{A. Meshing}

Meshing of the finite difference method is usually in manner of regular distribution to obtain the same form difference equation at each discrete point. The method is easy for the auto implementation of computer and can reduce computational complexity [6]. Among the three mode of meshing (square, triangle and hexagon), square meshing is often used.

For the actual billet specifications, the nodes and meshes are determined by the Fig.1. Assuming the heating and heat conduction of each side of the billet are the same in the induction heating process, the study object is simplified to $1 / 8$ of the billet cross-section (boxed part), includes 15 nodes.

\section{B. Determining the initial temperatures}

Since induction heating is an intermediate link of continuous casting direct rolling, it is unable to measure the internal temperature distribution of the billet first into the furnace. Finite element analysis software ANSYS is an effective tool to solve the coupling problem of the

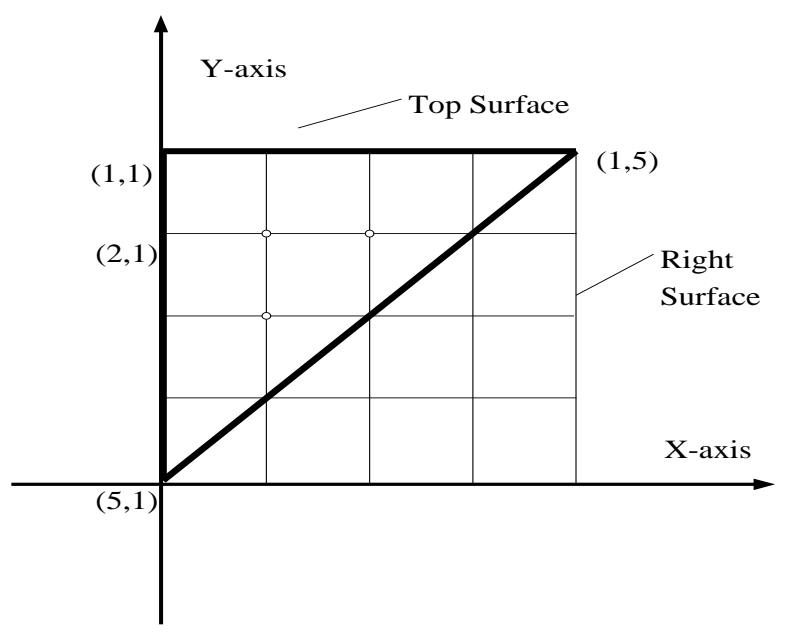

Figure 1. Two-dimensional structure diagram of the billet cross-section

two-dimensional structure diagram of the billet crosssection electromagnetic field and temperature field.

It is a common method to use ANSYS finite element analysis to simulate the billet induction heating process. But the defect is the computing time too long to meet the requirements of the online computing. The paper uses ANSYS to simulate the continuous induction heating process from the billet pulled from the mold to the end of induction heating. Through the obtained temperature distribution of the internal measuring points, not only the initial temperature is determined, but also the induction heating distributed parameter model based on the finite difference in the same heating condition is verified. The paper assumes that a billet is divided into $\mathrm{n}$ segments. The initial temperature of the billet nodes of the nth segment is determined by

$$
T^{n}(i, j, 0)=T_{0}^{n}(i, j)
$$

\section{Calculating eddy current power density $q_{v}$}

In the billet induction heating process, the internal heat is primary generated by eddy currents. The eddy current has an uneven distribution in the billet, declining exponentially with the increase distance to the surface of the billet. Thus, eddy power of each node is different.

The eddy current intensity of the point distant from the surface of the billet is

$$
I_{x}=I_{0} \exp (-x / \delta)
$$

Where $I_{0}$ is the eddy current intensity on the surface of the billet, $\mathrm{x}$ is the distance from the point to the surface of the billet, $\delta$ is skin depth.

Internal heat power generated by eddy currents exists in every node of the billet according to the (5).The discrete unit of one node is chosen to calculate, and its resistance is

$$
R=\frac{\rho l}{s}=\frac{\rho \Delta x}{d r \cdot \Delta z}
$$


Where $\rho$ is the resistance ratio of the billet, $\mathrm{r}$ is the distance from the node to the center of the billet, $\Delta x$ is the side length of a square mesh, $\Delta z$ is thickness of the billet. The eddy current induced on the discrete unit is

$$
\begin{aligned}
I & =I_{x} \cdot s=I_{0} \exp (-x / \delta) \cdot d r \cdot \Delta z \\
& =I_{0} \exp [(r-a / 2) / \delta] \cdot d r \cdot \Delta z
\end{aligned}
$$

Where $a$ is side length of the billet cross-section. According to the power computation formula, power integral factor of the discrete unit is

$$
\begin{aligned}
d P & =I^{2} \cdot R \\
& =I_{0}{ }^{2} \exp [(2 r-a) / \delta] \cdot d r \cdot \Delta z \cdot \rho \Delta x
\end{aligned}
$$

After integration and simplification, the paper gets

$$
\begin{aligned}
P_{i, j} & =\int_{r_{j-1}}^{r_{j}} d P=I_{0}^{2} \exp (-a / \delta) \cdot \Delta z \cdot \rho \Delta x \int_{r_{j-1}}^{r_{j}} \exp (2 r / \delta) d r \\
& =I_{0}^{2} \exp (-a / \delta) \cdot \Delta z \cdot \rho \Delta x \cdot \frac{\delta}{2}\left[\exp \left(2 r_{j} / \delta\right)-\exp \left(2 r_{j-1} / \delta\right)\right] \\
& =q_{v}
\end{aligned}
$$

Eddy power of any node in the discrete unit is obtained. The power allocated to the segment of the billet is the accumulated summation of the eddy currents of all the nodes. The paper assumes that the output power of the medium frequency power supply is averagely assigned to each segment of the billet as certain efficiency.

$$
P=\frac{k}{n} P_{0}=\sum_{i=1}^{n_{x}} \sum_{j=1}^{n_{y}} P_{i, j}
$$

Where $\mathrm{k}$ is the conversion efficiency of the output power, $\mathrm{n}$ is the number of the segment of the billet, $P_{0}$ is the output power of the power supply, $n_{x} 、 n_{y}$ is the number of the mesh in the direction of $\mathrm{X}, \mathrm{Y}$-axis. Thus, the eddy current on the surface of the billet is obtained by taking the (9) to the (10).

$$
I_{0}=\sqrt{\frac{2 k P_{0}}{n \cdot \Delta z \cdot \Delta x \sum_{i=1}^{n_{x}} \sum_{j=1}^{n_{y}} \rho \cdot \delta \cdot \exp (-a / \delta)\left[\exp \left(2 r_{j} / \delta\right)-\exp \left(2 r_{j-1} / \delta\right)\right]}}
$$

After the eddy current on the surface of the billet is obtained, the eddy current power, that is the heating rate density $q_{v}$, is obtained by (9).

\section{Finite difference}

The paper chooses $1 / 8$ of the billet cross-section as study object because of the symmetry of boundary conditions. The interval of the square meshes is $h$, $\Delta x=\Delta y=h$. As is shown in Fig. 2, the two-dimensional difference equation of the node $(i, j)$ of the nth segment of the billet is mainly established by heat conduction theory and energy conservation law. The temperature change of the node $(\mathrm{i}, \mathrm{j})$ after a time interval $\Delta \mathrm{t}$ from time $\mathrm{k}$ to time $\mathrm{k}+1$ is caused by the mutual effects of the heat transfer from the neighboring nodes $(i-1, j),(i+1, j),(i, j-1),(i, j+1)$ and the internal heat power generated by eddy currents.

With the change of temperature, the specific heat and thermal conductivity of the billet change correspondingly. The value of specific heat and thermal conductivity at different temperature is obtained by experimental measurements or tables. Then interpolation calculation based on the current temperature is used to get the specific heat and thermal conductivity value at current circumstance to make the model more accurate.

The paper assumes that the temperature of one certain node of the nth section of the billet at $\mathrm{k}$ different time points are $T^{n}(i, j, k)$, and its specific heat and thermal conductivity respectively are $c_{(i, j, k)}$ and $\lambda_{(i, j, k)}$.

The heat imported from node $(\mathrm{i}-1, \mathrm{j})$ is

$$
Q_{1}=\frac{\lambda_{(i, j, k)}\left(T^{n}(i-1, j, k)-T^{n}(i, j, k)\right) \cdot \Delta y}{\Delta x}
$$

The heat imported from node $(i+1, j)$ is

$$
Q_{2}=\frac{\lambda_{(i, j, k)}\left(T^{n}(i+1, j, k)-T^{n}(i, j, k)\right) \cdot \Delta y}{\Delta x}
$$

The heat imported from node $(\mathrm{i}, \mathrm{j}-1)$ is

$$
Q_{3}=\frac{\lambda_{(i, j, k)}\left(T^{n}(i, j-1, k)-T^{n}(i, j, k)\right) \cdot \Delta x}{\Delta y}
$$

The heat imported from node $(\mathrm{i}, \mathrm{j}+1)$ is

$$
Q_{4}=\frac{\lambda_{(i, j, k)}\left(T^{n}(i, j+1, k)-T^{n}(i, j, k)\right) \cdot \Delta x}{\Delta y}
$$

The internal energy power increment of node $(i, j)$ in time $\Delta \mathrm{t}$ is

$$
Q_{\Delta}=\frac{\rho c_{(i, j, k)}\left(T^{n}(i, j, k+1)-T^{n}(i, j, k)\right) \cdot \Delta x \cdot \Delta y}{\Delta t}
$$

According to energy conservation law:

$$
Q_{\Delta}=Q_{1}+Q_{2}+Q_{3}+Q_{4}
$$

Figure 2. Schematic diagram of node transfer 
The paper assumes that the temperature of one certain node of the nth section of the billet at $\mathrm{k}$ different time points are $T^{n}(i, j, k)$, and its specific heat and thermal conductivity respectively are $c_{(i, j, k)}$ and $\lambda_{(i, j, k)}$, the difference equation is :

$$
\begin{aligned}
& T^{n}(i, j, k+1)=T^{n}(i, j, k) \\
& +\frac{\Delta t \lambda_{(i, j, k)}}{\rho c_{(i, j, k)}}\left[\frac{T^{n}(i+1, j, k)+T^{n}(i-1, j, k)}{h^{2}}\right] \\
& +\frac{\Delta t \lambda_{(i, j, k)}}{\rho c_{(i, j, k)}}\left[\frac{T^{n}(i, j+1, k)+T^{n}(i, j-1, k)-4 T^{n}(i, n, k)}{h^{2}}\right]
\end{aligned}
$$

The skin effect and heat radiation on the surface of the billet considered, the difference equation is obtained:

$$
\begin{gathered}
T^{n}(1, j, k+1)=T^{n}(1, j, k) \\
+\frac{\Delta t \lambda_{(1, j, k)}}{\rho c_{(1, j, k)}}\left[\frac{T^{n}(2, j, k)+T^{n}(1, j-1, k)+T^{n}(1, j+1, k)}{h^{2}}\right] \\
-\frac{\Delta t \lambda_{(1, j, k)}}{\rho c_{(1, j, k)}} \frac{3 T^{n}(1, j, k)}{h^{2}}+\frac{\Delta t q_{v} k_{2}}{\rho c_{(1, j, k)}}-\frac{\Delta t q_{r}}{\rho c_{(1, j, k)}} \\
T^{n}(1,5, k+1)=T^{n}(1,5, k)+\frac{\Delta t \lambda_{(1, j, k)}}{\rho c_{(1, j, k)}}\left[\frac{2 T^{n}(1,4, k)-2 T^{n}(1,5, k)}{h^{2}}\right] \\
+\frac{\Delta t q_{v} k_{2}}{\rho c_{(1, j, k)}}-\frac{2 \Delta t q_{r}}{\rho c_{(1, j, k)}}
\end{gathered}
$$

E. Results verification

Some coefficients should be adjusted appropriately in the equations and the results are compared with the data of ANSYS simulation to get a conclusion.

\section{SimUlation RESULTS AND ANALYSIS}

\section{A. Actual production environment}

In this paper, the production line of the billet induction heating is shown in Fig. 3. The heating means are formed by the two sensors. Each sensor is 1meter long. The space between the sensors is $1.6 \mathrm{~m}$. The infrared thermometer sensor for temperature detection before heating is 0.4 meters away from the inlet of the first sensor. The infrared thermometer sensor for temperature detection after heating is 0.6 meters away from the outlet of the second sensor. The size of the billet is $0.15 \mathrm{~m} \times 0.15 \mathrm{~m} \times 6 \mathrm{~m}$, and is divided into 120 segments. Roller drive speed is $0.2 \mathrm{~m} / \mathrm{s}$, the total heating time is 23 seconds. The time interval of temperature detection is 0.5 second, so there are 46 temperature detections in the heating process

\section{B. Simulation steps.}

According to the (8), (9) and (10), the temperature distribution of each node in each segment of the billet during the heating is obtained. The paper creates an induction heating model in MATLAB. The steps are:

(1) Field data including the voltage, current and frequency of the induction heating power supply is collected, and the current of the induction coil according to the principle of circuit design is calculated;

(2) Through the finite element simulation of billet induction heating in ANSYS, simulated temperature distribution of the billet induction heating process is obtained.

(3) The Finite element meshes of the billet are generated, and a distributed parameter model of the billet induction heating is established. Then the model is calculated in MATLAB.

\section{Example analysis}

The $80^{\text {th }}$ segment of the billet is taken as an example. The Fig. 4 shows the initial temperature distribution of the billet cross-section. The Fig. 5 shows that the ANSYS simulation data is consistent to the distributed parameter model results after $14 \mathrm{~s}$ heated by the first sensor. It also shows that there is a significant rise on the surface of the billet, and the closer the node to the surface of the billet the faster the temperature promoted. The center temperature of the billet changes little in a short time, since it is dominated by thermal conduction. The Fig. 6 shows that, the ANSYS simulation data is also consistent to the distributed parameter model results after the billet pulled out of furnace, The final billet surface temperature has been increased from $840 \sim 880{ }^{\circ} \mathrm{C}$ to $1000 \sim 1160{ }^{\circ} \mathrm{C}$, but due to the soaking time is short, the cross section temperature difference is about $100{ }^{\circ} \mathrm{C}$.

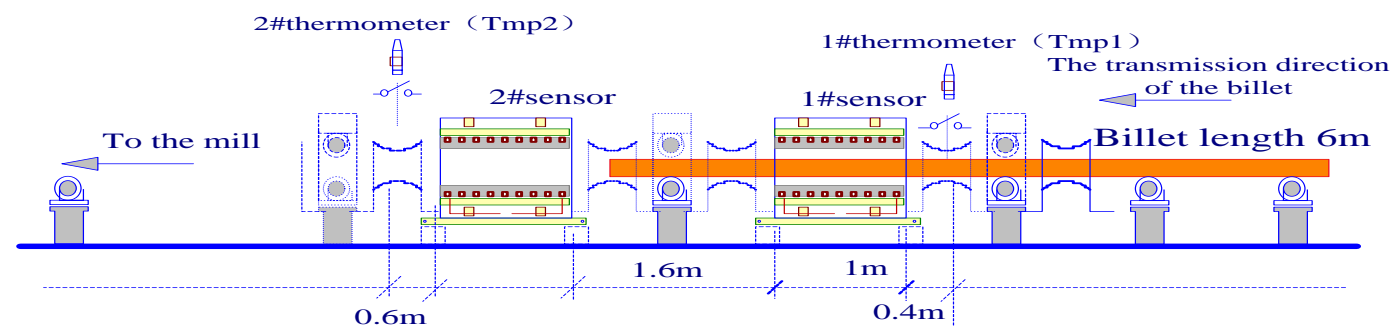

Figure 3. Induction heating billet production line 


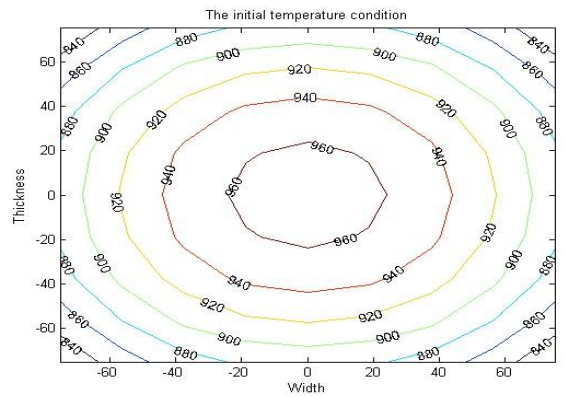

Figure 4. The initial temperature distribution of the billet cross-section
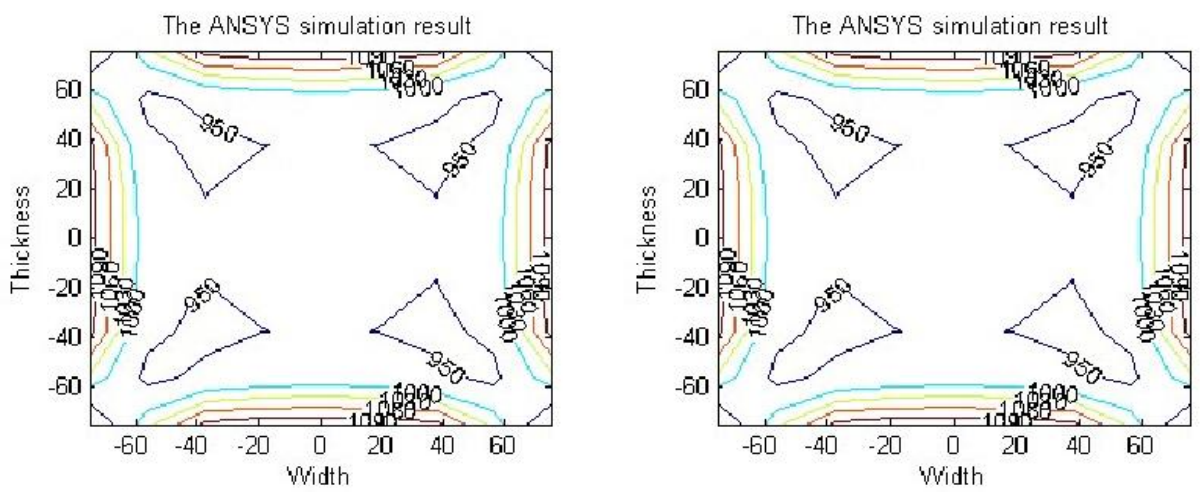

Figure 5. Temperature distributions comparison after 14 seconds billet heating
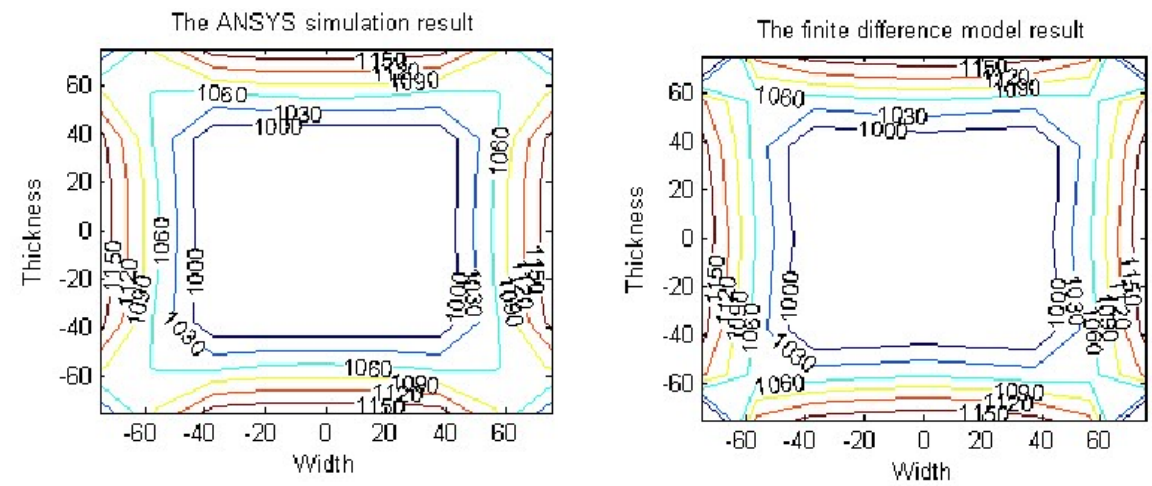

Figure 6. Temperature distributions comparison after the billet out of furnace

The $80^{\text {th }}$ segment of the billet is also taken as an example to study a single node. The results are shown in Fig. 7-10. They are respectively temperature distribution of the surface node $(1,2)$, two internal node $(2,2)$ and $(3,2)$, and the central node $(5,1)$. The figure shows that, there is not heat radiation in the central node, the resulting eddy current could not reach, either. Temperature changes mainly depend on temperature heat conduction, yet heat conduction in a short time due to small temperature changes. From the previous three nodes, it was found that there are two phases of temperature rising exactly when the billet is heating in the sensor. Before the induction heating, the surface temperature is apparently decreased due to the heat radiation, while compared with the surface the internal node temperature drops marginally because there is only heat conduction. In order to determine model is appropriate more intuitively, the error rate of the above 4 nodes were calculated the equation of the error rate $\mathrm{P}$ is defined as follow:

$$
P=\left[\frac{1}{n} \sum_{i=1}^{n}\left(q_{i}-\tilde{q}_{i}\right)^{2}\right]^{1 / 2} / \bar{q}
$$

Where $q_{i}$ is the ANSYS simulation data (representing the measured value); $\tilde{q}_{i}$ is the prediction value; $\bar{q}$ is the arithmetic average of the ANSYS simulation data; $n$ is the 


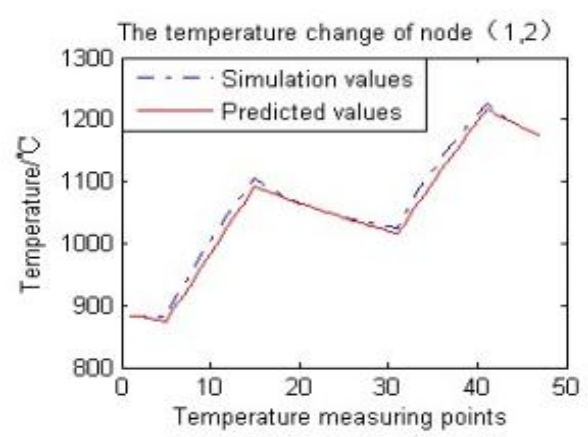

Figure 7. Temperature change of node $(1,2)$

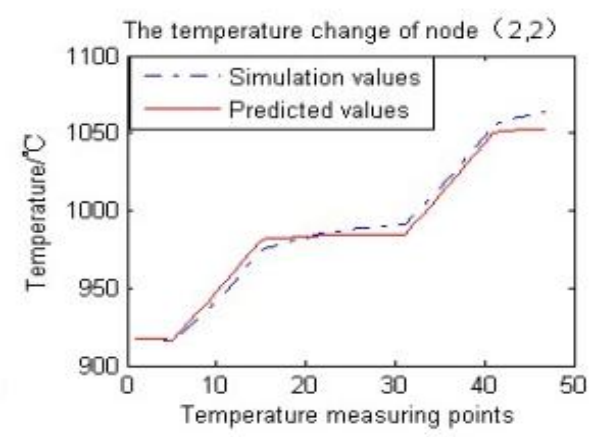

Figure 8. Temperature change of node $(2,2)$

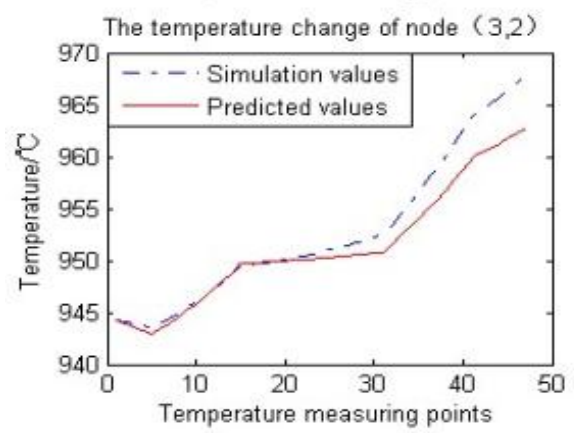

Figure 9. Temperature change of node $(3,2)$

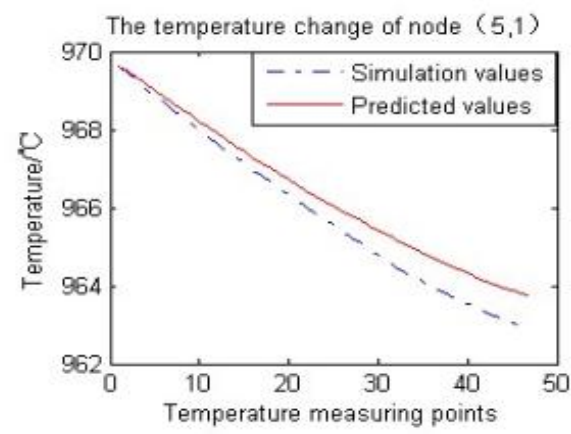

Figure 10. Temperature change of node $(5,1)$ length of the data.

The prediction errors of the four nodes are $1.1 \%, 0.5 \%$, $0.2 \%$ and $0.1 \%$, the average error of all nodes is $2.57 \%$. The results show that the distributed parameter model is basically consistent with ANSYS simulation. Because of the ANSYS simulation with characteristics of high precision, the billet temperature predicted by the distributed parameter model is also of high precision. In the calculation of time, it takes the ANSYS software about 10 minutes to calculate the induction heating process of each segment of the billet through the finite element method, while solving the finite difference model of distributed parameter by MATLAB programming needs only 20 seconds.

\section{SUMMARY}

A finite element model established in ANSYS is a common method to simulate the continuous casting billet induction heating process. It is of high precision, but the computing time is longer, unable to meet the demand of online computing. The distributed parameter model based on the finite difference of the billet induction heating established in this paper can predict the temperature distribution of billet cross-section of each segment quickly. The results are basically consistent with simulated data in ANSYS. It shows that both the prediction accuracy and the computing speed of the model are high. Therefore the model can achieve a goal of accurate prediction and control

\section{REFERENCES}

[1] B.S. Jovanovic, L.G. Vulkov, On the convergence of finite difference schemes for the heat equation with concentrated capacity. Numerische Mathematik, 2001,89(4), pp 715-734.

[2] Xue Qiong, Xiao Xiaofeng, Realization of finite volume method of two-dimensional heat conduction equation in MATLAB, Chinese Computer Engineering and Applications, 2012,48 (24) , 197-221.

[3] Liu Hao, Chen Liliang, Zhou Jianxin, Numerical simulation of billet induction heating temperature field based on ANSYS, Chinese Special Casting \& Nonferrous Alloys, 2007,27(4), 259-261.

[4] Ting Wang, New techniques for designing a finite difference domain decomposition algorithm for the two- and three-dimensional heat equations. International Journal of Computer Mathematics, 2007, 84(7), pp989-1002.

[5] F. Asllanaj; A. Milandri; G. Jeandel; J. R. Roche, A finite difference solution of non - linear systems of radiative-conductive heat transfer equations. International Journal of Numerical Methods In Engineering, 2002, 54(11), pp1649-1668.

[6] Li Wangshan, Wang Wenqia, Two-dimensional heat equation of finite difference domain decomposition algorithm, Chinese Jouranl of ShangDong University, 2011,46 (12) ， 1-12 